\title{
Shocking the brain to regain motor function: a non-invasive therapy for stroke patients
}

\author{
Michael Min Wah Leung
}

\begin{abstract}
Invasive treatments and its associated risks are important factors of concern when the conditions are affecting the nervous system. Transcranial direct current stimulation (tDCS) is a non-invasive technique that stimulates brain areas through the scalp and has excitatory or inhibitory neuromodulatory effects. In the context of stroke patients, recovery is often impaired from the increased inhibition of the damaged area from the unaffected hemisphere. Fujimoto et al. uses dual-hemisphere transcranial direct current stimulation to address this interhemispheric inhibition and demonstrates that stroke patients were able to periodically restore sensory deficits.
\end{abstract}

Keywords: Transcranial direct current stimulation; Stroke; Dual-hemisphere tDCS; Parkinson's Disease

\section{Résumé}

Les traitements invasifs et ses risques associés nécessitent une attention particulière lorsque les conditions affectent le système nerveux. La stimulation transcrânienne à courant continu (tDCS) est une technique non invasive qui stimule certaines régions du cerveau à travers la scalpe soit de façon excitatrice ou de façon neuromodulante inhibitrice. Dans le contexte de patients souffrant d'un ACV, leur rétablissement est souvent compromis à cause de l'inhibition accrue de la région endommagée de l'hémisphère non affectée. Fujimoto et al. utilisent une tDCS à hémisphère double afin de résoudre cette inhibition inter-hémisphérique et démontrent les déficits sensoriels ont été rétablis de façon périodique que chez les patients souffrant d'un ACV.

Mots Clés: Stimulation transcrânienne à courant direct; Accident vasculaire cérébral; tCDS à double hémisphère; La maladie de Parkinson

The preference of minimally invasive treatment to remedy various disorders and conditions is gaining popularity in patient care, emphasizing safety, recovery, and comfort. The advancements in minimally invasive treatments are essential for conditions associated with the nervous system, as high risks are normally associated with neurological medical intervention (2). Transcranial direct current stimulation (tDCS) is a non-invasive technique that can alter cellular activity using weak electric currents (3). The efficacy of tDCS depends on the method of delivery, and the trending procedure in most recent studies is by dual-hemisphere tDCS.

Dual-hemisphere tDCS makes use of interhemispheric interactions to optimize behavioral performance, in which one hemisphere is excited while the other is inhibited (4). This methodology of simultaneously using

Correspondence: mleun036@uottawa.ca

Department of Cellular and Molecular Medicine, University of Ottawa, 451 Smyth Rd, K1H 8M5, Ottawa, Canada

The Ottawa Hospital Research Institute, 725 Parkdale Avenue, K1Y 4E9, Ottawa, Canada excitatory (anodal) and inhibitory (cathodal) signals with cathodes placed in opposite or less affected areas is well-documented (2-7). Fujimoto and colleagues have reported that a single session of dual-hemisphere tDCS over the primary and secondary somatosensory areas resulted in a transient behavioral gain in tactile discrimination with stroke patients. To support their theory, the authors compiled results from 8 applicable chronic stroke patients who all suffered a supratentorial stroke and exhibit sensory deficits with a Mini Mental Status Examination score of more than 24 points. This experiment was the first double-blind, sham-controlled experimental study for dual-hemisphere dTCS, and measured tactile discrimination via performance of both index fingers in the grating orientation task (GOT) before, during, and after dual-hemisphere tDCS over the primary and secondary somatosensory cortices (S1 and S2, respectively) (4).

Each patient was subject to the GOT while blindfolded and in a comfortable position. Tactile stimuli from plastic domes varying in groove widths were applied onto the 


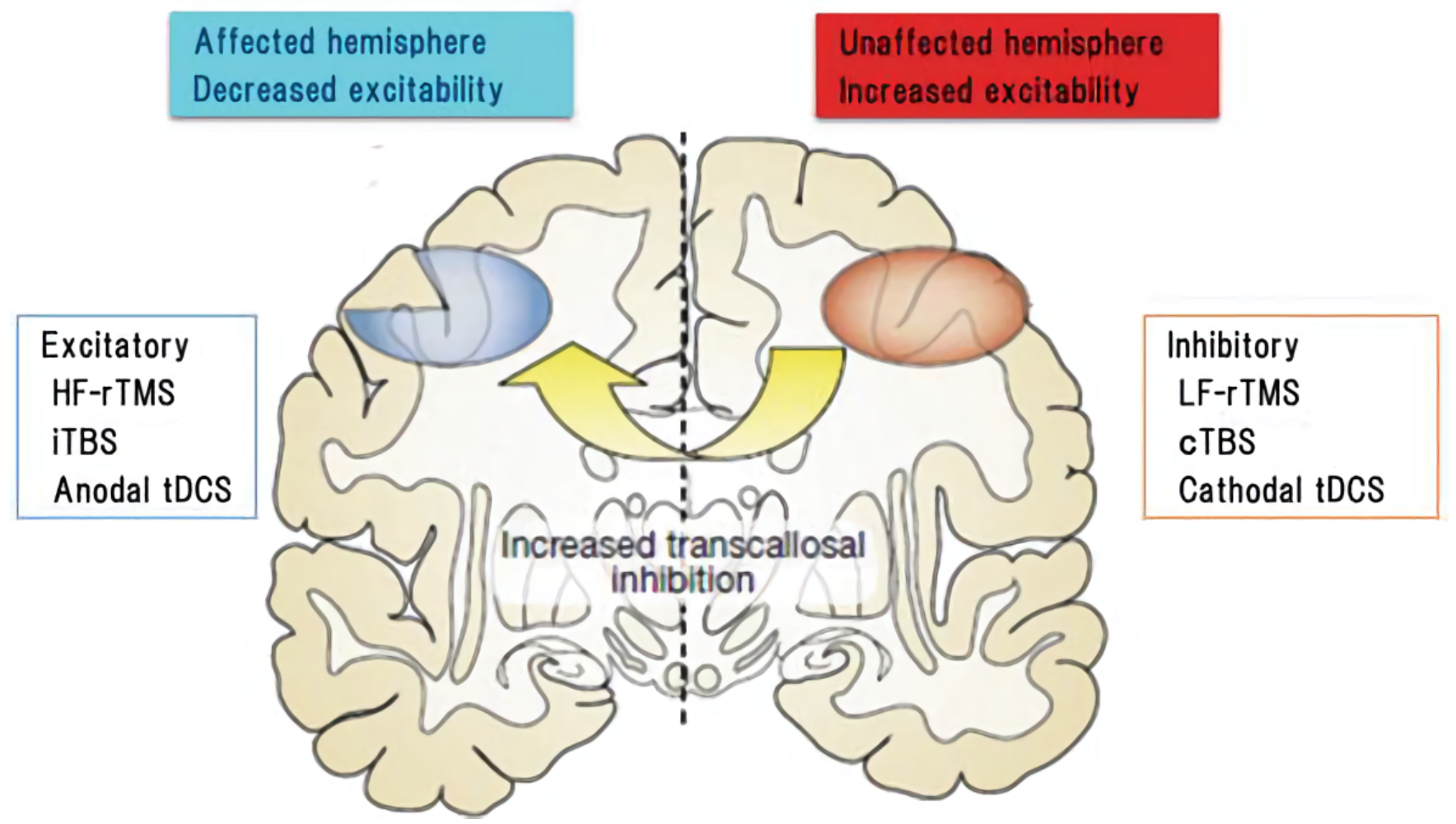

Figure 1: Interhemispheric interactions schematic from Matsuda et al., 2017. (1)

patient's palmar side of the affected and non-affected index fingers for two seconds, and they had to differentiate the orientation of the groove (parallel or orthogonal to the axis of the index finger). The authors evaluated each patient's performance by assigning a threshold score, calculated by the finest grating spacing each patient could discern with their index finger. This experiment was repeated three times (pre-intervention, intra-intervention, and post-intervention) and was done again with the nonaffected index finger. To identify the regions over the primary and secondary somatosensory cortices (S1 and S2), the researchers used magnetic resonance imaging. A verbal rating was also collected from patients in case there was any subjectivity impacting their performance.

Dual-hemisphere tDCS over the primary and secondary somatosensory cortices helped improve patient performance in the GOT. Fujimoto and colleagues claim that the behavioral gain observed was enhanced by decreased activity in the unaffected hemisphere, reducing interhemispheric inhibition during the task. Results from the GOT gathered from each patient supported their hypothesis, as the experimental stimulations in both somatosensory cortexes allowed all participants to discriminate finer crevices with their affected index finger (4).

Although Fujimoto et al. hypothesized that a single session of dual-hemisphere tDCS is more effective than single hemisphere tDCS, their current study had not shown comparable data between single hemisphere tDCS and dual-hemisphere tDCS. If single hemisphere tDCS was sufficient to elicit the same behavioral gain, then the use of dual-hemisphere tDCS was redundant. The author's original hypothesis that dual-hemisphere tDCS enhanced tactile discrimination in stroke patients in both the S1 and $\mathrm{S} 2$ regions was supported by their data. However, a judgment could not be made as to which somatosensory cortex was a more suitable target for tDCS due to the similar behavioral gain in S1 and S2 stimulations. Furthermore, each patient's elicited behavioral gain was of different magnitude due to the heterogeneity of each patient's stroke localization (4). Although the heterogeneity exhibited practicality in patients with different stroke severity and localization all benefited from tDCS, future avenues of study should explore homogenous pathologies, so conclusions could be drawn.

The future in the field of minimally invasive therapeutic treatments is expanding, with tDCS gaining popularity for its ease of use and access. Furthermore, Fujimoto and colleagues were successful in demonstrating improved somatosensory function in stroke patients via dual-hemisphere tDCS over S1 and S2. Alternative therapeutic treatments to tDCS include DBS (deep brain stimulation) and rTMS (repetitive transcranial magnetic stimulation), but tDCS remains the preferred treatment option due to a favourable safety profile, tolerability, eas- 
ier applicability and cost effectiveness (5). Previous studies demonstrate the efficacy of tDCS to improve motor function in neurological patients suffering with sensory and motor deficits, such as cerebellar ataxia, supratentorial stroke, and Parkinson's disease (2-7). Future longitudinal studies could aid stroke patients with long term rehabilitation, with beneficial effects on somatosensory performance.

Competing interests

The author declares that he has no competing interests.

References

1. T. Matsuda, A. Manji, K. Amimoto, A. Inaba, Y. Wada, Non-Invasive Brain Stimulation (TMS/tDCS) and Rehabilitation for Stroke and Parkinson's (InTechOpen, 2017), chap. 8, pp. 121-135.

2. J. V. Rosenfeld, Aust New Zeal J Surg 66, 553 (1996).

3. N. G. Pozzi, et al., Cerebellum 13, 109 (2014).

4. S. Fujimoto, et al., Front Neurosci 10, 128 (2016).

5. D. H. Benninger, et al., J. Neurol. Neurosurg. Psychiatry 81, 1105 (2010).

6. A. Fusco, et al., Stroke Res Treat 2013, 837595 (2013).

7. D. Impey, S. de la Salle, V. Knott, Brain Cogn 105, 46 (2016). 